\title{
Light-induced behavioural effects on the locomotor activity rhythm of the blow fly, Calliphora vicina (Diptera: Calliphoridae)
}

\author{
DAVID S. SAUNDERS ${ }^{1}$ and BRONISLAW CYMBOROWSKI ${ }^{2 *}$ \\ ${ }^{1}$ Emeritus Professor, University of Edinburgh, UK \\ ${ }^{2}$ Department of Animal Physiology, University of Warsaw, Poland; e-mail: bron@biol.uw.edu.pl
}

Key words. Circadian, blow fly, locomotor activity, continuous light

\begin{abstract}
Under continuous light (LL) of low intensity (up to 2.0 lux), the free-running period of the blow fly activity rhythm lengthened for flies whose initial $\tau_{\mathrm{DD}}$ was less than about $25 \mathrm{~h}$, but shortened for longer-period flies. Light intensity above about 2.0 lux led to an overt behavioural arrhythmicity. A comparison of phases of the oscillation after LL as a function of phases before LL, however, indicated that the oscillation continued its motion even though overt behaviour was arrhythmic. As the intensity during LL increased to 48 lux, phases after LL became centred on a narrow range close to CT 12. Extended periods of brighter light are considered to have two effects: (a) an exogenous "masking" effect occurring at intensities greater than about 2.0 lux, and (b) at intensities between about 2.0 and 48 lux, a gradual resetting of the covert oscillator to CT 12. Between 2.0 and 48 lux, therefore, the circadian oscillation governing locomotor activity in C. vicina continues its motion despite the superficial arrhythmicity in behaviour.
\end{abstract}

\section{INTRODUCTION}

In many insects, a transfer from continuous darkness (DD) to continuous light (LL) of low intensity causes the circadian period $(\tau)$ to lengthen; transfer to LL of a higher intensity then frequently results in behavioural arrhythmicity (Konopka et al., 1989; Hong \& Saunders, 1994; Saunders, 2002, for references). With females of the dayactive blow fly Calliphora vicina, for example, $\tau$ increased from about $22.5 \mathrm{~h}$ in DD to about $25.6 \mathrm{~h}$ in LL up to 20 lux, with locomotor behaviour becoming arrhythmic at light intensities much above that value (Hong \& Saunders, 1994). A similar phenomenon has also been observed for eclosion rhythmicity. Working with Drosophila pseudoobscura, Winfree (1974) demonstrated a lengthening of $\tau$ in very dim light, likened to "starlight", but arrhythmicity occurring in brighter conditions, which he likened to "moonlight". Data such as these form part of a general observation dubbed "Aschoff's rule" (Saunders, 2002).

Coupled with observations such as these is the finding (e.g. in Drosophila pseudoobscura; Pittendrigh, 1966) that an extended period of moderately bright light (of at least 10 to $12 \mathrm{~h}$ duration) appears to "dampen out" the rhythm of pupal eclosion, only for it to restart at a phase equivalent to the beginning of the subjective night (CT 12) when - or soon after - the light goes out (Pittendrigh, 1966). An important question arising from these long light-related phenomena concerns the state of the rhythm and its underlying circadian oscillation during the period of extended illumination. Is the observed behavioural arrhythmicity a result of exogenous "masking" by the light, or is the motion of the underlying oscillation brought to a standstill? In an earlier paper (Hong \& Saun- ders, 1994) this problem was not resolved. The question is now addressed with further observations on the rhythm of locomotor activity in the blow fly Calliphora vicina.

\section{MATERIALS AND METHODS}

Flies

The strain of Calliphora vicina $\mathrm{R} .-\mathrm{D}$. used in this investigation was isolated near Warsaw, Poland $\left(52^{\circ} \mathrm{N}\right)$ in 2003 . Maintenance of stock cultures was as described earlier (Saunders, 1987; Cymborowski, 1998).

\section{Recording locomotor activity}

A $5 \mathrm{~cm}$ diameter Petri dish was glued to the centre of a larger, $9 \mathrm{~cm}$, Petri dish to form a circular track. The inner dish contained cotton wool, soaked in sugar solution, with some of the cotton drawn through small lateral holes to provide a source of sugar and water for the fly. A single male fly was placed within the track. Two such Petri dishes were mounted on a wooden platform which also provided support for the infra-red emitters (Radio Spares type 306-077) and detectors (RS type 306-083). An infra-red light beam was arranged to pass vertically across the side of the circular track containing the fly. The whole assembly was then enclosed in a light-tight wooden box held in a constant temperature room at $26 \pm 1^{\circ} \mathrm{C}$. Each box was provided with a $4 \mathrm{~W}$ fluorescent lamp. At the level of the experimental fly, irradiance from this lamp was about 48 lux unless reduced light intensities were used (see below).

\section{Reduction in light intensity}

The reduced light intensities used in this study were achieved by wrapping paper of various thicknesses around the fluorescent tubes, with both ends of the tubes tightly wrapped in aluminium foil to prevent leakage of light. Reduced light intensities were measured with a JU 16 photometer in lux. The range of light intensities achieved by this method was from about 1.5 lux to about 48 lux.

\footnotetext{
* Corresponding author.
} 


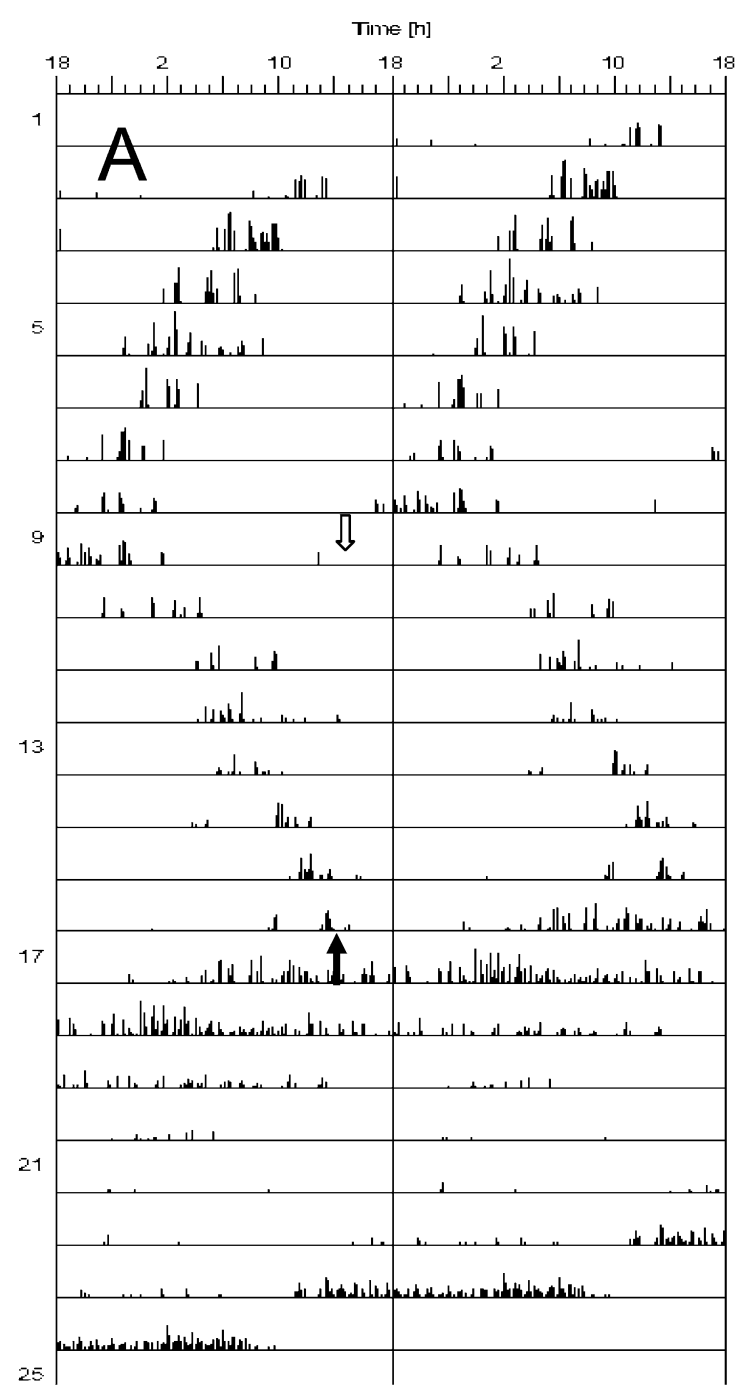

\section{Experimental design}

Male flies within $24 \mathrm{~h}$ of emergence were placed in the recording devices in continuous darkness (DD) for 7 days to record their initial free-running activity rhythms. They were then exposed to constant light (LL) of various intensities for up to 7 days before a final free-run in DD. In different experiments the times of transfer from DD to LL, and from LL to DD, were systematically varied to occur at $2 \mathrm{~h}$ intervals (local time).

TABLE 1. Proportions of flies with rhythmic and arrhythmic locomotor activity in constant light of various intensities.

\begin{tabular}{lccc}
\hline Light intensity in LL & $\mathrm{N}$ & No. rhythmic (\%) & No. arrhythmic (\%) \\
\hline $1.5 \operatorname{lux}$ & 53 & $47(88.7 \%)$ & $7(13.2 \%)$ \\
$0.0022 \mathrm{~W} \mathrm{~m}^{-2}$ & & & \\
$2.0 \operatorname{lux}$ & 51 & $25(49.0 \%)$ & $26(51.0 \%)$ \\
$0.0029 \mathrm{~W} \mathrm{~m}^{-2}$ & & & \\
$12 \operatorname{lux}$ & 23 & $3(13.0 \%)$ & $20(86.9 \%)$ \\
$0.0175 \mathrm{~W} \mathrm{~m}^{-2}$ & & & $22(95.7 \%)$ \\
$24 \operatorname{lux}$ & 23 & $1(4.3 \%)$ & $59(100 \%)$ \\
$0.035 \mathrm{~W} \mathrm{~m}^{-2}$ & & & \\
$48 \operatorname{lux}$ & 59 & $0(0 \%)$ & \\
$0.07 \mathrm{~W} \mathrm{~m}^{-2}$ & & &
\end{tabular}
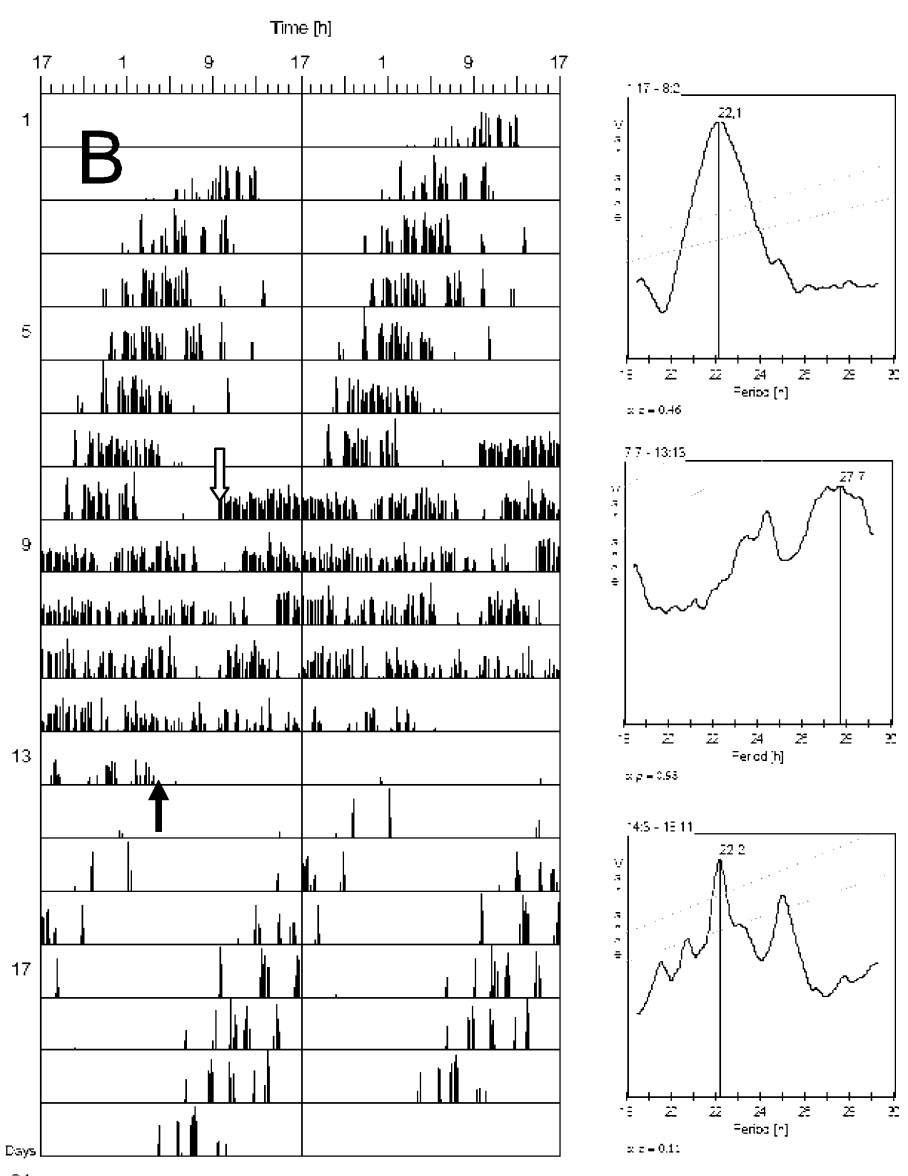

$==-0 .+6$

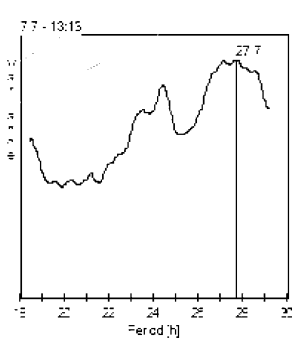

$\because F=\hat{x}$. S

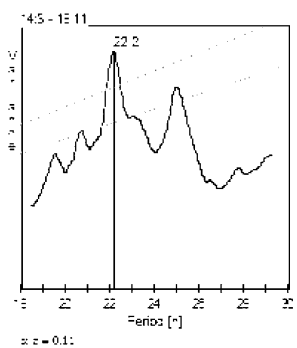

Fig. 1. Representative locomotor activity rhythms of adult male Calliphora vicina at $26^{\circ} \mathrm{C}$. A - fly initially in continuous darkness (DD), then exposed to continuous light (LL) of low intensity (2.0 lux), and finally back to DD, showing period lengthening in LL. B - fly exposed to a higher intensity light (48 lux) during LL, showing behavioural arrhythmicity.

Activity events were registered as the number of times the moving fly broke the infra-red light beam within successive 10-min intervals. These numbers were recorded using a PC and assembled into the conventional "double-plotted" format (Kenny \& Saunders, 1991). Free-running periods $(\tau)$ were calculated, either by periodogram analysis (based on Enright, 1965, and Williams \& Naylor, 1978) or by eye-fitting a line through the mid-points of each day's activity band, a phase considered to represent Circadian Time (CT) 06 (Cymborowski et al., 1993).

\section{Calculations of phase shifts}

Transfers of flies from DD to LL were arranged to occur at 2 $\mathrm{h}$ intervals throughout the day; later transfers back from LL to DD occurred at the same times for each fly. Phase (circadian time) of the DD to LL transfer for each fly was calculated using the time of transfer and the period of the fly's activity rhythm leading up to that transfer. In that way, a range of onset phases was achieved. The phase of the rhythm after transfer from DD to LL or from LL to DD was calculated by extrapolating back to the transfer using, once again, the period of the oscillation to determine circadian times. Phase shifts were then calculated, in circadian hours, using the mid-point of the activity band (CT 06) as a phase reference point, the phase shift data being 
TABLE 2. Circadian periods of rhythmic flies in DD and LL, showing percentage lengthening in $\tau$ after transfer to continuous light (LL) and back to darkness (DD). Only those flies that remained rhythmic in LL are included.

\begin{tabular}{|c|c|c|c|c|c|c|}
\hline $\begin{array}{l}\text { Light intensity } \\
\text { In LL }\end{array}$ & $\mathrm{N}$ & Initial $\tau_{\mathrm{DD}} \mathrm{h} \pm 2 \mathrm{SE}$ & $\tau_{\mathrm{LL}} \mathrm{h} \pm 2 \mathrm{SE}$ & Change in $\tau \%$ & Final $\tau_{\mathrm{DD}} \mathrm{h} \pm 2 \mathrm{SE}$ & Changein $\tau_{\mathrm{DD}} \%$ \\
\hline $\begin{array}{l}1.5 \operatorname{lux} \\
0.0022 \mathrm{Wm}^{-2}\end{array}$ & 41 & $24.35 \pm 0.480$ & $24.86^{\mathrm{a}} \pm 0.509$ & $2.42 \%$ & $25.59^{\mathrm{a}} \pm 0.460$ & $0.99 \%$ \\
\hline $\begin{array}{l}2.0 \operatorname{lux} \\
0.0029 \mathrm{Wm}^{-2}\end{array}$ & 20 & $23.60 \pm 0.828$ & $24.96^{\mathrm{a}} \pm 0.600$ & $5.72 \%$ & $23.86^{\mathrm{a}} \pm 1.214$ & $1.10 \%$ \\
\hline $\begin{array}{l}12 \operatorname{lux} \\
0.0175 \mathrm{Wm}^{-2}\end{array}$ & 3 & $21.77 \pm 1.729$ & $26.70 \pm 0.982$ & $23.90 \%$ & $22.23 \pm 1.841$ & $2.16 \%$ \\
\hline $\begin{array}{l}24 \operatorname{lux} \\
0.035 \mathrm{Wm}^{-2}\end{array}$ & 1 & 22.20 & 26.70 & $20.27 \%$ & 27.20 & \\
\hline
\end{tabular}

${ }^{\mathrm{a}} \mathrm{p}<0.05$

1.5 lux: $\tau_{\mathrm{LL}} \mathrm{v} \tau_{\mathrm{DD}}$ initial, $\mathrm{df}=40, \mathrm{t}=-2.3584, \mathrm{p}<0.05 ; \tau_{\mathrm{DD}}$ final $\mathrm{v} \tau_{\mathrm{DD}}$ initial, $\mathrm{df}=40, \mathrm{t}=-0.9491, \mathrm{p}<0.05$

2.0 lux: $\tau_{\mathrm{LL}} \mathrm{v} \tau_{\mathrm{DD}}$ initial, $\mathrm{df}=19, \mathrm{t}=-3.0241 . \mathrm{p}<0.05 ; \tau_{\mathrm{DD}}$ final $\mathrm{v} \tau_{\mathrm{DD}}$ initial, $\mathrm{df}=19, \mathrm{t}=-0.6743, \mathrm{p}<0.05$

expressed as "old phase-new phase" or phase transition curves (PTCs) (Johnson et al., 2004).

\section{RESULTS}

\section{Effects of continuous light (LL) on locomotor rhythmicity}

During their initial free-runs in continuous darkness, the flies' circadian periods $\left(\tau_{\mathrm{DD}}\right)$ varied widely from 19.2
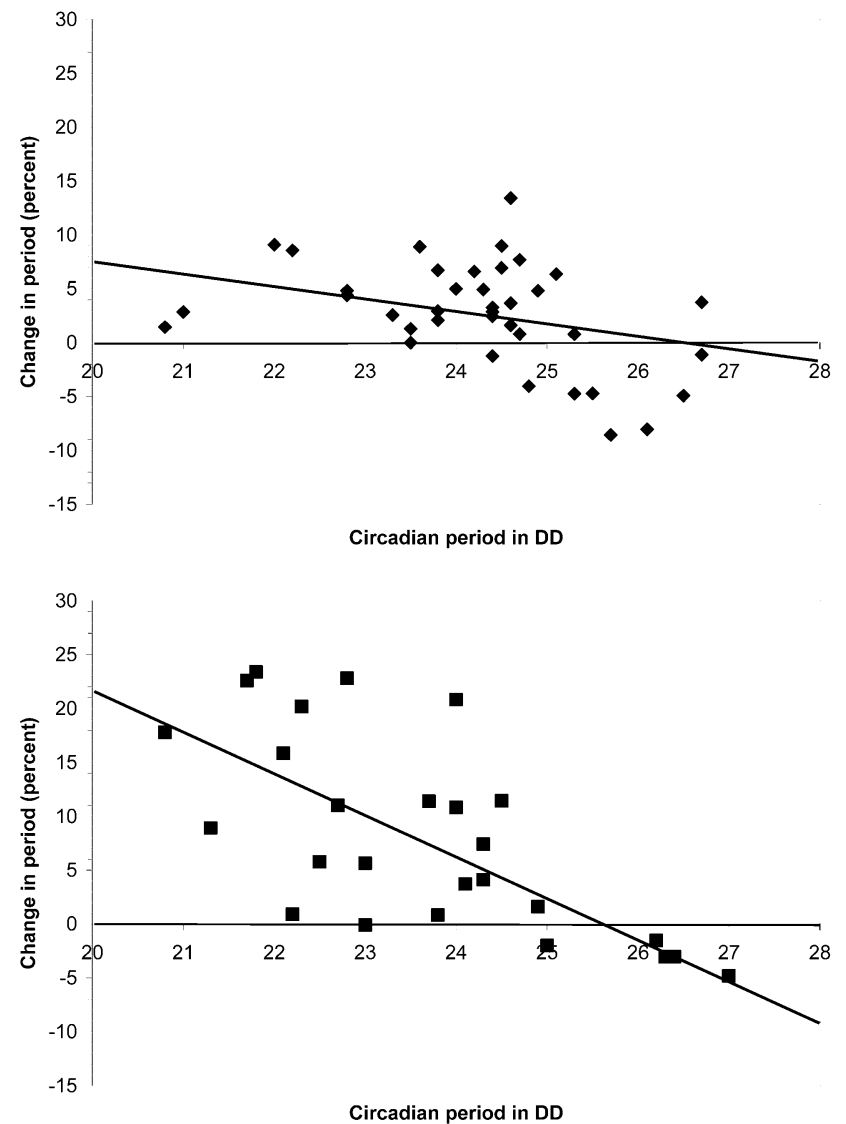

Fig. 2. The change in period during LL as a function of the initial period in darkness. Upper panel: LL at 1.5 lux; lower panel: LL at 2 lux. Flies with an initial $\tau_{\mathrm{DD}}$ shorter than about 25 $\mathrm{h}$ show a period lengthening; those with $\tau_{\mathrm{DD}}$ greater than about $25 \mathrm{~h}$ show a slight period shortening. to as much as $30.6 \mathrm{~h}$, with mean values in different experimental groups varying from 22.16 to $24.69 \mathrm{~h}$ (data not shown). The onset of light and subsequent exposure to LL then caused either (1) a change in $\tau$, or (2) behavioural arrhythmicity, depending upon light intensity (Fig. 1).
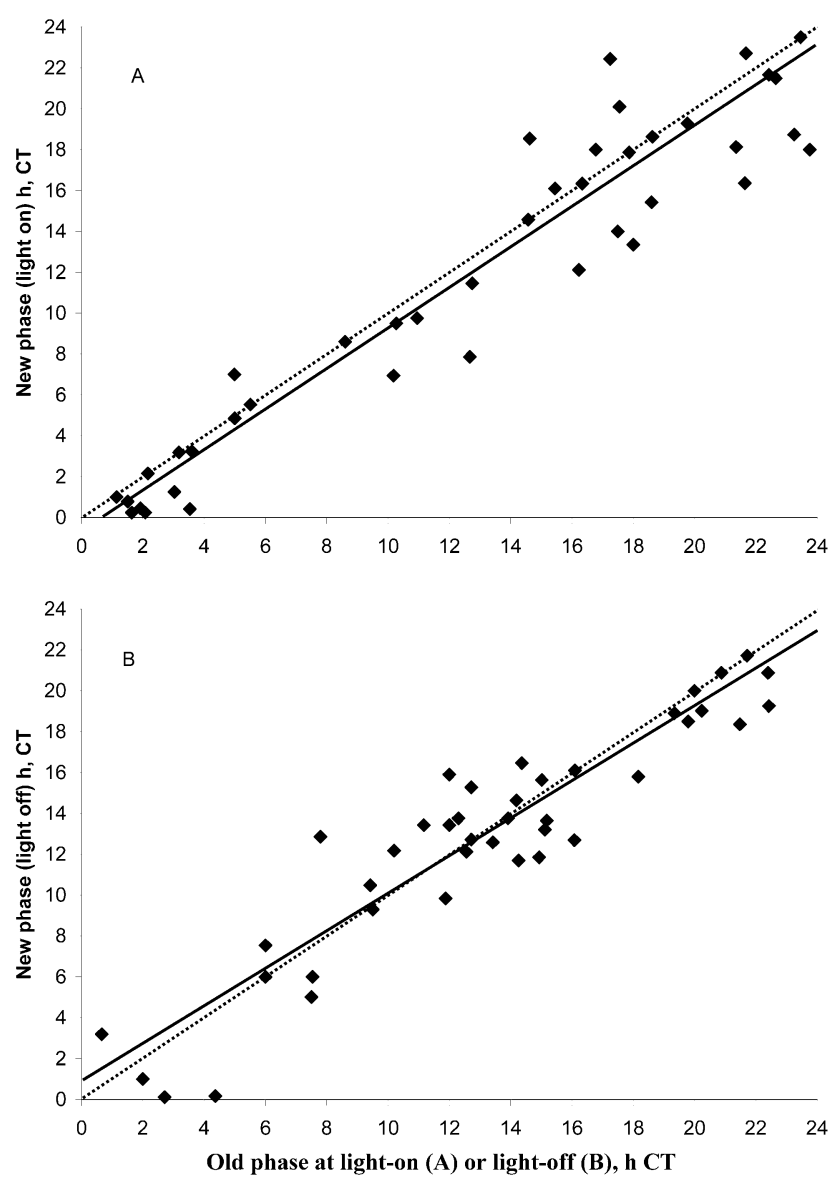

Fig. 3. Phase transition curves (PTCs) showing the transition from old phase to new phase at light-on (panel A) or at light-off (panel B). Light intensity during LL, 1.5 to 2.0 lux. Solid lines show regressions, $\mathrm{A}$ : $\mathrm{y}=-0.418+0.952 \mathrm{x} ; \mathrm{B}: \mathrm{y}=0.787+$ $0.914 \mathrm{x}$. Dotted lines show new phase $=$ old phase slopes of 1.0 , indicating that the experimental PTCs were of Type 1. 

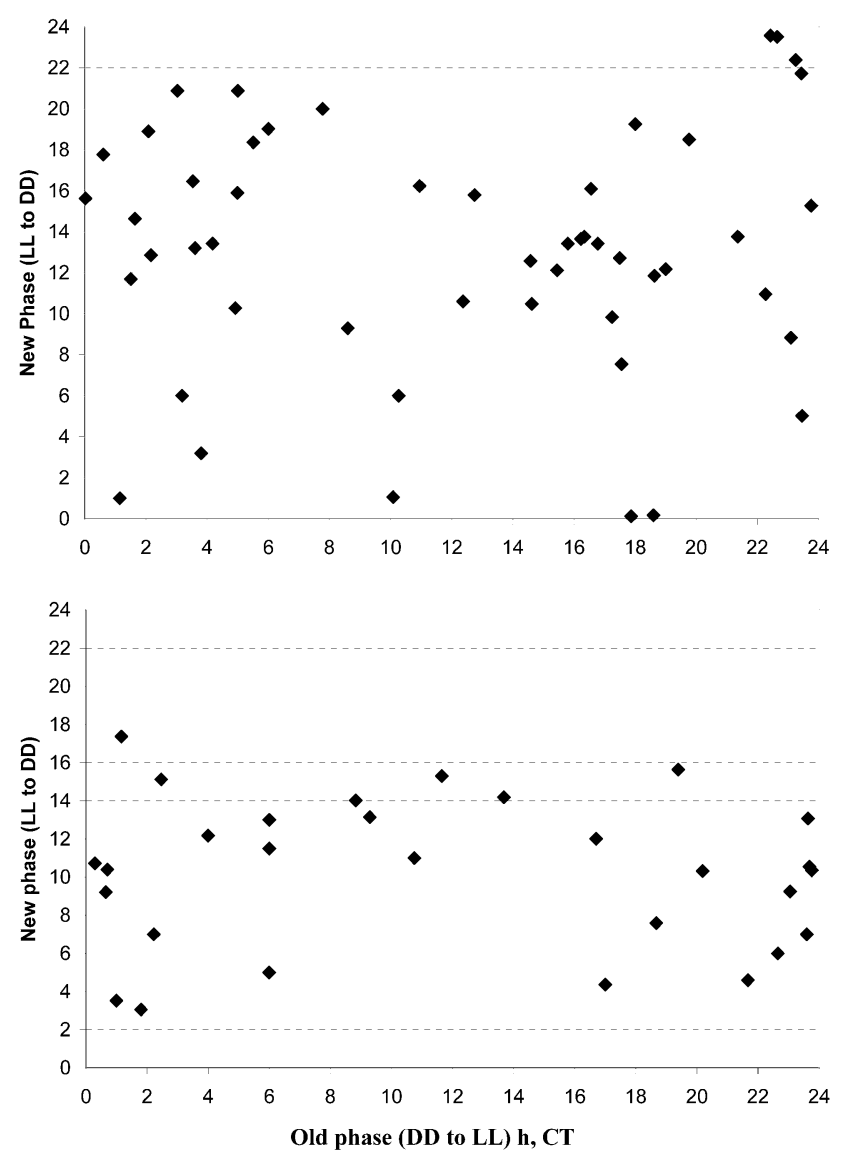

Fig. 4. Phase transition responses of flies exposed to an extended time under weak LL (1.5 to 2.0 lux) comparing flies that remained rhythmic under LL (upper panel) with those that became arrhythmic (lower panel). The wide scatter of data in the lower panel indicates that the oscillators persist during LL, even though overt behaviour becomes arrhythmic.

Table 1 shows that only a minority (about 13 per cent) of the flies became arrhythmic under LL of the lowest intensity tested (1.5 lux). This proportion then rose to about 50 per cent under 2.0 lux, and to nearly 90 per cent under 12 lux. Exposure to the brightest light tested (48 lux) caused arrhythmicity in all of the flies. The threshold intensity for arrhythmic behaviour was therefore close to 2.0 lux for males of this particular strain of $C$. vicina maintained under the experimental conditions used in this investigation.

Table 2 shows the effect of light intensity on $\tau_{\mathrm{LL}}$ in those flies that remained rhythmic under constant light. Under 1.5 lux, $\tau_{\mathrm{LL}}$ generally increased by 2.4 per cent over their $\tau_{\mathrm{DD}}$ values; this lengthening became 5.7 per cent under 2.0 lux and rose to more than 20 per cent in the few flies that remained rhythmic under 12 and 24 lux. Flies that remained rhythmic under constant light showed a wide range of $\tau_{\mathrm{LL}}$ values from 21.1 to $29.0 \mathrm{~h}$. These data show that light intensities below that leading to arrhythmic locomotor behaviour caused a change in circadian period, most commonly shown as a lengthening of $\tau$.
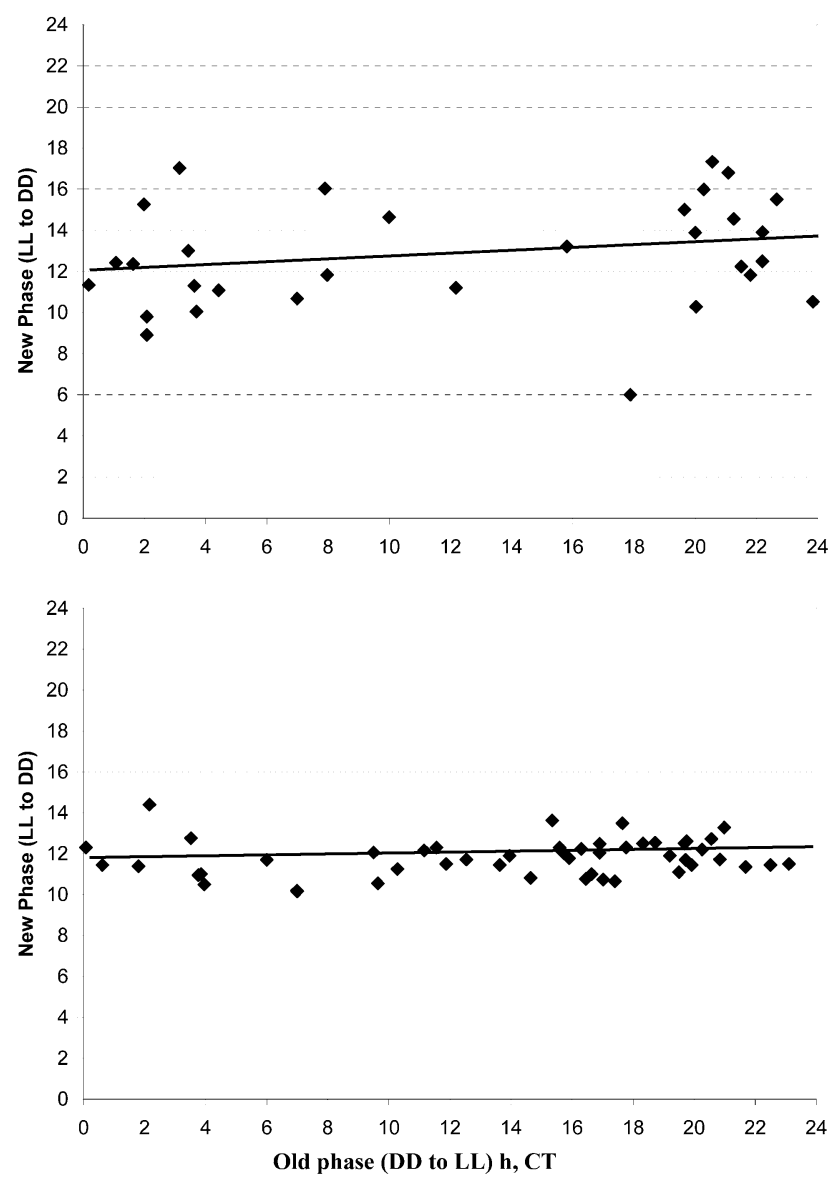

Fig. 5. Phase transition curves for arrhythmic flies exposed to LL of a higher intensity. Upper panel: 12 to 24 lux; lower panel: 48 lux. Data show a narrowing of new phase distribution (after LL) to a near-constant phase approaching CT 12, indicating a Type 0 PTC. Upper panel: regression $\mathrm{y}=11.938+0.069 \mathrm{x}$; lower panel $\mathrm{y}=11.521+0.021 \mathrm{x}$.

Table 2 also shows that flies moving from LL at 1.5 lux into a final period under DD showed an additional slight lengthening of $\tau$, but those moving from LL at 2.0 lux into DD showed a slight shortening. A similar final shortening was observed in the 3 flies moving from LL at 12 lux into DD.

Although, on average, exposure to LL caused period lengthening, this was not always the case. Fig. 2 shows that period lengthening occurred mainly with shorter period flies, but those with initial $\tau_{\mathrm{DD}}$ values of $25 \mathrm{~h}$ or more frequently showed a shortening of their endogenous period after transfer to LL. For example, Fig. 2 (upper panel) shows that 6 of the 8 flies whose initial $\tau_{\mathrm{DD}}$ values were greater than $25 \mathrm{~h}$ showed such period shortening under LL of 1.5 lux, and all 5 of those with $\tau_{\mathrm{DD}}$ greater than $25 \mathrm{~h}$ did so at 2.0 lux (Fig. 2, lower panel).

Phase resetting effects of low intensity illumination at light-on and light-off

Transfer of flies from DD to low intensity (1.5 to 2.0 lux) LL at $2 \mathrm{~h}$ intervals across the day, coupled with their very wide range of initial $\tau_{\mathrm{DD}}$ values, led to an equally wide range of circadian phases at the onset of the light. This allowed for the construction of a phase transition 
curve (PTC) for the single step-wise transition from darkness to light (Fig. 3, upper panel). In flies that remained rhythmic under these conditions, and therefore free-ran throughout the period in LL, a similar PTC for the stepwise transfer from light to the final period of darkness could also be constructed (Fig. 3, lower panel). In both cases the phase shifts were small, giving rise to "Type 1" PTCs with an average slope close to 1 (Fig. 3) (light on: linear regression: $\mathrm{y}=-0.418+0.952 \mathrm{x}, \mathrm{n}=41, \mathrm{r}^{2}=0.9065$; light off: $\mathrm{y}=0.787+0.914 \mathrm{x}, \mathrm{n}=41, \mathrm{r}^{2}=0.8354$ ). Therefore, despite the fact that these transitions from darkness to light, or from light to darkness, caused substantial changes in $\tau$, any phase shifts generated were small or sometimes non-existent.

Phase resetting effects of extended periods of constant illumination in rhythmic and arrhythmic flies exposed to low intensity $L L$ (1.5 to 2.0 lux)

Fig. 4 plots the circadian phases of flies at the end of an extended period of low intensity light (LL, 1.5-2.0 lux) as a function of their phases at its inception, comparing those flies that remained rhythmic (upper panel) with those that became arrhythmic under LL (lower panel). In this analysis, only those flies that retained clear rhythmicity after exposure to LL could be used; consequently the number of flies (data points) appearing in Fig. 4 are fewer than those appearing in Table 1. Such old phase-new phase plots produced a very wide scatter of new phases. In the case of rhythmic flies (Fig. 4, upper panel) this was a "full" range from about CT 0 to CT 24 at light off; in the arrhythmic flies the scatter was almost as wide, from about CT 3 to CT 18.

\section{Phase resetting effects of $L L$ at higher intensities (12 to 48 lux)}

Effects of exposures to LL of higher light intensity are shown in Fig. 5, with 12 to 24 lux in the upper panel, and 48 lux in the lower. The majority of the flies in these groups were arrhythmic under constant light. Under LL of 12 to 24 lux, the scatter of phases at light off was less wide than at 1.5 to 2.0 lux (Fig. 4, lower panel), ranging from about CT 6 to CT 18. Under the brightest light tested (48 lux) this reduction was continued with a much smaller scatter of phases, from CT 10 to CT 14 (Fig. 5, lower panel). In Fig. 5, both sets of data showed phase transition curves with linear regressions for the phases at light-off close to CT $12(12-24$ lux: $y=11.938+0.069 x$, $\mathrm{n}=31 ; 48$ lux: $\mathrm{y}=11.521+0.021 \mathrm{x}, \mathrm{n}=49)$. These data thus suggest that with increasing light intensity, an extended period of light brings all phases toward CT 12 at light-off, giving rise to a "Type 0" PTC.

\section{DISCUSSION}

\section{The effects of LL on $\tau$ and arrhythmicity}

The present results show that $\tau_{\mathrm{DD}}$ values for males of Calliphora vicina were highly variable, from about 19 to $30 \mathrm{~h}$. The very short period flies and very long period flies may be natural variants that resemble, for example, $\mathrm{per}^{S}$ and $\mathrm{per}^{l}$ mutants of Drosophila melanogaster
(Konopka \& Benzer, 1971), although it is not known whether the innate periods of these flies are heritable.

Transfer of $C$. vicina males from DD to LL caused an alteration in $\tau$, generally an increase, or the onset of behavioural arrhythmicity, with a threshold between $\tau$ increase and arrhythmicity close to 2 lux. This observation parallels that obtained earlier for females of $C$. vicina from a stock isolated in Scotland (Hong \& Saunders, 1994). The LL-induced increase in $\tau$ violates "Aschoff' $s$ rule" which (for day-active vertebrates) suggests that a transfer from DD to LL shortens $\tau$ and that increased light intensity shortens $\tau$ still further (Menaker, 1968; Aschoff, 1979, 1981). This conclusion is consistent with the view that "Aschoff's rule" is not generally applicable to insects, nearly every species of which shows a lengthening of $\tau$ upon transfer to constant light, regardless of whether it is considered to be diurnally or nocturnally active (Hong \& Saunders, 1994; Saunders, 2002, for references).

The results presented here, however, show that the relationship between LL and $\tau$ is not straightforward. In $C$. vicina, flies with an initial $\tau_{\mathrm{DD}}$ value of $25 \mathrm{~h}$ or less, generally show a $\tau$ lengthening, whereas those with a longer value of $\tau_{\mathrm{DD}}$ may show period shortening. This observation may be compared with data for the period mutants of D. melanogaster (Konopka et al., 1989). In this study, short period $\left(\mathrm{per}^{S}\right)$ and long period $\left(\mathrm{per}^{L}\right)$ flies were exposed to constant light for 10 days following 10 days in DD. Under LL of low intensity (0.001 to 0.1 lux) the periods of 6 out of $9 \mathrm{per}^{S}$ flies shortened still further, whereas 7 out of $11 \mathrm{per}^{L}$ flies lengthened. At intensities above 0.1 lux the periods of both genotypes lengthened. These results thus differ from those with $C$. vicina in the present experiments, in which the period in constant light tended to lengthen for shorter period flies but shorten for those with a longer period.

\section{The phase resetting effects of an extended period under $\mathbf{L} \mathbf{L}$}

Comparisons of phases before and after a time in LL provide information on the behaviour of the circadian oscillators during constant light. At the lowest light intensities tested (1.5 to $2.0 \mathrm{lux}$ ) some of the flies remained rhythmic in LL, albeit with altered periodicity, whereas others became behaviourally arrhythmic. The rhythmic flies (Fig. 4, upper panel) displayed a very wide range of phases at the end of LL as a result of the variation of phases at the onset of LL combined with the wide range of $\tau_{\mathrm{LL}}$ values during illumination itself. In the arrhythmic flies under the same conditions of illumination (Fig. 4, lower panel) this scatter of phases was almost as great, suggesting very strongly that the individual oscillators were persisting during LL with their own $\tau_{\mathrm{LL}}$ values, but the above-threshold light caused behavioural arrhythmicity by exogenous or positive "masking".

As the light intensity during LL increased from 2.0 to 48 lux, the range of phases at the LL to DD transition ("new phases") steadily narrowed until all were close to CT 12 at the light-off signal (Fig. 5). This suggests that 
"beneath" the light-induced masking effect of brighter light, the individual oscillators persisted for a while, perhaps with decreased amplitude, until almost coming to a standstill close to CT 12. In this respect, the behaviour of locomotor rhythmicity in the diurnally-active species $C$. vicina closely resembles that of pupal eclosion in the crepuscular Drosophila pseudoobscura (Pittendrigh, 1966) although much brighter light of longer duration is required in the blow fly.

\section{Exogenous and endogenous effects of $L L$ on locomotor} rhythmicity in $C$. vicina

The present results show that extended periods of illumination have two effects on the rhythm of locomotor activity in $C$. vicina. The first of these is an exogenous "masking" effect on activity leading to overtly arrhythmic behaviour of the fly when the light (LL) exceeds 2 lux. As in similar such masking effects, this arrhythmic activity ceases abruptly as soon as the period of illumination ceases. The second effect is one on the endogenous circadian system itself, firstly changing the free-running period from its original value in DD - and then as light intensity increases and is on for a sufficient length of time - bringing the circadian oscillation to a halt at a phase close to CT 12 . Under the considerably brighter levels of illumination pertaining in the natural environment, it is likely that daily photophases in the order of 8 to $12 \mathrm{~h}$ will also bring the oscillation to that phase so that the onset of darkness occurs at the beginning of the subjective night (CT 12). Both the exogenous and endogenous effects of LL must contribute to the rhythmicity of locomotor activity in the natural environment.

It is likely that the two observed affects of LL on locomotor rhythmicity - "masking" and bringing the oscillator to rest at CT 12 - use different light input pathways. In an earlier paper (Cymborowski et al., 1994) it was shown that bilateral optic lobectomy, which effectively isolates the compound eyes from the brain, left the phenomenon of entrainment intact. This suggests that the circadian oscillator controlling locomotor activity is in the central brain, and that entraining light reaches this oscillator by direct access through the brain tissues. On the other hand, Cymborowski et al. (1994) also observed that unoperated or sham operated flies frequently showed some locomotor activity after the end of the entraining light phase which was absent in lobectomised flies. The increased activity immediately following light off in control flies and its absence in lobectomised flies suggests that this activity is a direct or "masking" effect of light via the eyes and optic lobes, similar to that described for the eclosion rhythm in the silkmoth Hyalophora cecropia (Truman, 1972). The entraining effects of light on the circadian oscillator may thus be direct to the brain, whereas "masking" effects may operate via the compound eyes.

ACKNOWLEDGEMENTS. This work was supported in part by Ministry of Sciences and Higher Education, Poland, Grant No: N303 068 31/2338 to B. Cymborowski.

\section{REFERENCES}

Aschoff J. 1979: Circadian rhythms: influences of internal and external factors on the period measured in constant conditions. Z. Tierpsychol. 49: 225-249.

Aschoff J. 1981: Freerunning and entrained circadian rhythms. In Aschoff J. (ed.): Handbook of Behavioral Neurobiology. Vol. 4. Biological Rhythms. Plenum Press, New York, pp. 81-93.

Cymborowski B. 1998: Serotonin modulates a photic response in circadian locomotor rhythmicity of adults of the blow fly, Calliphora vicina. Physiol. Entomol. 23: 25-32.

Cymborowski B., Gillanders S.W., Hong S.-F. \& Saunders D.S. 1993: Phase shifts of the adult locomotor activity rhythm in Calliphora vicina induced by non-steroidal ecdysteroid agonist RH 5849. J. Comp. Physiol. (A) 172: 101-108.

Cymborowski B., Lewis R.D., Hong S.-F. \& Saunders D.S. 1994: Circadian locomotor activity rhythms and their entrainment to light-dark cycles continue in flies (Calliphora vicina) surgically deprived of their optic lobes. J. Insect Physiol. 40: 501-510.

ENRIGHT J. 1965: The search for rhythmicity in biological timeseries. J. Theor. Biol. 8: 426-468.

Hong S.-F. \& SAUNDERS D.S. 1994: Effects of constant light on the rhythm of adult locomotor activity in the blowfly, Calliphora vicina. Physiol. Entomol. 19: 319-324.

Johnson C.H., Elliott J., Foster R., Honma K.-I. \& Kronauer R. 2004: Fundamental properties of circadian rhythms. Chapter 3. In Dunlap J.C., Loros J.J. \& DeCoursey P.J. (eds): Chronobiology: Biological Timekeeping. Sinauer Associates, Sunderland, pp. 67-105.

KenNY N.A.P. \& SAunders D.S. 1991: Adult locomotor rhythmicity as "hands" of the maternal photoperiodic clock regulating larval diapause in the blowfly, Calliphora vicina. $J$. Biol. Rhythms 6: 217-233.

KonopKa R. \& Benzer S. 1971: Clock mutants of Drosophila melanogaster. Proc. Nat. Acad. Sci. USA 68: 2112-2116.

Konopka R., Pittendrigh C.S. \& OrR D. 1989: Reciprocal behaviour associated with altered homeostasis and photosensitivity of Drosophila clock mutants. J. Neuro-Genet. 6: 1-10.

MENAKER M. 1968: Extraretinal light perception in the sparrow. I Entrainment of the biological clock. Proc. Nat. Acad. Sci. USA 59: 414-421.

PitTeNDRIgH C.S. 1966: The circadian oscillation in Drosophila pseudoobscura pupae: a model for the photoperiodic clock. $Z$. Pflanzenphysiol. 54: 275-307.

SAUNDERS D.S. 1987: Maternal influence on the incidence and duration of larval diapause in Calliphora vicina. Physiol. Entomol. 12: 331-338.

SAUNDERS D.S. 2002: Insect Clocks. $3^{\text {rd }}$ ed. Elsevier, 560 pp.

TRUMAN J.W. 1972: Circadian rhythms and physiology with special reference to neuro-endocrine processes in insects. In Bierhuizen J.F. (ed.): Circadian Rhythmicity. Centre for Agricultural Publishing and Documentation, Wageningen, pp. $111-135$.

Williams J.A. \& NAYLoR E. 1978: A procedure for the assessment of significance of rhythmicity in time-series data. Int. J. Chronobiol. 5: 435-444.

WinfreE A.T. 1974: Suppressing Drosophila's circadian rhythm with dim light. Science 183: 970-972.

Received January 30, 2008; revised and accepted April 9, 2008 